\title{
GAPS IN THE ESSENTIAL SPECTRUM FOR SECOND ORDER SYSTEMS
}

\author{
ROBERT M. KAUFFMAN
}

ABSTRACT. Consider the equation $\left(D^{2}+A+a E\right) f=0$, where $a$ is a positive real number, $E$ is the $n \times n$ identity matrix, $A$ is a continuously differentiable function from $[0, \infty)$ to the $n \times n$ Hermitian matrices, and $A$ and $A^{\prime}$ are bounded. It is shown that, if $\alpha$ is large with respect to $\|A\|_{\infty}$, there are small positive numbers $\lambda$ such that, for every solution $f$ to the equation $\left(D^{2}+A+a E\right) f=0, e^{-\lambda t} f(t)$ is square integrable, but $e^{\lambda t} f(t)$ is not. It is also shown that, if $a$ is large with respect to $\|A\|_{\infty}$, there is a real number $\lambda$ close to zero such that $\lambda$ is in the essential spectrum of any selfadjoint operator in $L_{2}$ associated with $D^{2}+A+a E$. These results generalize the results of Hartman and Putnam, who proved these statements for the scalar case $n=1$.

The equation $y^{\prime}+A(t) y=0$, where $A$ is a continuous function from $[0, \infty)$ to the $n \times n$ Hermitian matrices, occurs often in mechanics. If $A(t)$ is periodic, this equation is equivalent, by a periodic change of variables, to the equation $z^{\prime}=R z$, where $R$ is a constant $2 n \times 2 n$ matrix. If $R$ has no multiple eigenvalues, and if all eigenvalues have a nonzero real part, it follows that range $L_{0}$ is closed, where $L=D^{2}+A$, and $D=d / d t$. Thus, if $R$ has no multiple eigenvalues, and range $L_{0}$ is not closed, and $A$ is periodic, it follows that there exist bounded solutions to the equation $L y=$ 0 . (In the scalar case, every solution is bounded.) Thus it is of interest to know when range $L_{0}$ is closed.

Even if range $L_{0}$ is closed, we can attempt to calculate the rate of growth of solutions. We study both these questions in this paper. Our results are not limited to the case where $A$ is periodic, but work whenever $A$ and $A^{\prime}$ are bounded.

The results may be stated as follows. If $A$ is a bounded function from $[0, \infty)$ to the $n \times n$ Hermitian matrices, with $A$ continuously differentiable and $A^{\prime}$ bounded, let $\|A\|_{\infty}$ denote $\operatorname{lub}\{|A(t)|: t \in[0, \infty)\}$, where for any

Received by the editors June 7, 1974 .

AMS (MOS) subject classifications (1970). Primary 34C10, 34B25.

Key words and phrases. Rate of exponential growth, essential spectrum, selfadjoint operator, Fredholm operator. 
$n \times n$ matrix $B,|B|^{2}=\operatorname{lub}\{\langle B x, B x\rangle /\langle x, x\rangle\}$, with $\langle$,$\rangle denoting the usual$ inner product in $C^{n}$. Let $K_{A}=\operatorname{glb}\left\{\|A-\lambda E\|_{\infty}: \lambda \in[0, \infty)\right\}$, where $E$ is the $n \times n$ identity matrix. Then, if $\|A\|_{\infty}$ is very large with respect to $K_{A}$, there are very small real numbers $\lambda$ such that $(L+\lambda E)_{0}$ does not have closed range. Furthermore, if $\|A\|_{\infty}$ is large with respect to $K_{A}$, there are very small positive real numbers $\lambda$ such that, for every $f$ with $L f=0$, $e^{\lambda t}|f(t)|$ is not in $L_{2}[0, \infty)$, but $e^{-\lambda t}|f(t)|$ and $e^{-\lambda t}\left|f^{\prime}(t)\right|$ converge to zero as $t$ approaches infinity, where, for $v \in C^{n},|v|=(\langle v, v\rangle)^{1 / 2}$. How large $\|A\|_{\infty}$ needs to be with respect to $K_{A}$ depends only on how large $\left\|A^{\prime}\right\|_{\infty}$ is. Precise estimates are contained in the results.

For the one dimensional case, when $A$ is a real valued function, these results, with somewhat different estimates and quite different methods of proof, were obtained by Hartman and Putnam [3]. Their methods do not appear to extend to the case of a second order system.

We recapitulate some known results about differential operators.

Notation. $L_{2}^{n}[0, \infty)$ denotes the space of measurable functions $f$ from $[0, \infty)$ into complex $n$ dimensional space $C^{n}$ such that $\langle f, f\rangle$ is in $L_{1}[0, \infty)$, where $\langle$,$\rangle denotes the dot product in C^{n} . L_{2}^{n}[0, \infty)$ is clearly a Hilbert space, with inner product denoted by $($,$) .$

Notation. Throughout the paper, let $S$ denote the set of all $f$ in $L_{2}^{n}[0, \infty)$ such that $D f$ is absolutely continuous and $D^{2} f$ is in $L_{2}^{n}[0, \infty)$, where $D=d / d t$. It is well known that for $f \in S, D f \in L_{2}^{n}[0, \infty)$.

Definition. Let $L=D^{2}+\lambda D+B$, where $\lambda$ is a real number, and $B$ be a bounded continuous function from $[0, \infty)$ to the $n \times n$ complex Hermitian matrices. Let $L_{M}$ denote the restriction of $L$ to $S . L_{M}$ is called the maximal operator associated with $L$.

Notation. Let $L$ be as in the previous definition. Then $L^{+}$denotes the expression $D^{2}-\lambda D+B$.

Notation. Let $S_{0}$ denote the set of all $f$ in $S$ such that $f(0)=D f(0)=$ 0 .

Definition. Let $L$ be as in the above definitions. Let $L_{0}$ denote the restriction of $L$ to $S_{0} . L_{0}$ is called the minimal operator associated with $L$.

Theorem 1. Suppose that $L=D^{2}+\lambda D+B$, where $\lambda$ is real and $B$ is a continuous, bounded function from $[0, \infty)$ to the complex $n \times n$ Hermitian matrices. Then $\left(L_{0}\right)^{*}=\left(L^{+}\right)_{M}$.

Notation. Let $L_{R}$ denote the restriction of $L$ to $C_{0}^{\infty}(0, \infty)$. 
Theorem 2. Let $L$ be as in Theorem 1. Then $L_{0}$ is the closure of $L_{R}$.

Remark. Both of these theorems are well known in the scalar case, and seem to be fairly well known in the present context, although the proofs in the matrix case may not be easily accessible in print. The general case can be proved fairly easily by using the scalar case, or alternatively by using the methods used, in the scalar case, by Dunford and Schwartz [1]. The following theorem was proved by Kauffman [4]. It follows fairly easily from the fact that $S$ is a $2 n$ dimensional extension of $S_{0}$.

Theorem 3. Suppose that range $L_{0}$ is closed, where $L$ is as in Theorems 1 and 2. Then nullity $L_{M}+$ nullity $\left(L^{+}\right)_{M}=2 n$.

Definition. A Fredholm operator is a closed linear operator with closed range, finite nullity, and finite deficiency. The index of a Fredholm operator is its nullity minus the deficiency of its range.

Theorem 4. Suppose $T$ is a Fredholm operator. Suppose that domain B contains domain $T$, where $B$ is a closed linear operator. Then there is $a$ $\delta>0$ such that, if $|\lambda|+|\alpha|<\delta$, index $(T+\lambda B+\alpha I)=\operatorname{index} T$, where $I$ is the identity operator, and where $T, B$ and $I$ act in $L_{2}^{n}[0, \infty)$.

Theorem 4 is a special case of [2, Theorem V.3.6, p. 122] since, by Theorem V.3.6, $B$ is $T$ bounded.

Notation. Throughout this paper, $I$ denotes the identity operator in $L_{2}^{n}[0, \infty)$.

We are now ready to begin our main results.

Notation. Let $A$ be as above. Then $K_{A}$, as before, denotes glb $\left\{\|A-\lambda E\|_{\infty}: \lambda \in[0, \infty)\right\}$, with $E$ the $n \times n$ identity matrix.

Theorem 5. Suppose tbat $A$ is a continuously differentiable function from $[0, \infty)$ to the $n \times n$ complex Hermitian matrices. Suppose that both $A$ and $A^{\prime}$ are bounded. Let $\delta$ be a positive real number. Then, if $\|A\|_{\infty}+$ $\delta^{2}>\left(\left\|A^{\prime}\right\|_{\infty} / 2 \delta\right)+2 K_{A}$, it follows that, for every solution $f$ to the equation $\left(D^{2}+A\right) f=0, e^{-\delta t} f(t)$ and $e^{-\delta t} f^{\prime}(t)$ are in $L_{2}^{n}[0, \infty)$ and converge to zero as $t$ approaches infinity, but $e^{\delta t} f(t)$ is not in $L_{2}^{n}[0, \infty)$.

Proof. For any real $\lambda$, let the differential expression $Q_{\lambda}$ be defined by $Q_{\lambda}=D^{2}+2 \lambda D+A+\lambda^{2} E$. Note that, for any twice differentiable $f, Q_{\lambda} f$ $=e^{-\lambda t}\left(D^{2}+A\right)\left(e^{\lambda t} f\right)$. Also, $\left(Q_{\lambda}\right)^{+} Q_{\lambda}=\left(D^{2}+A+\lambda^{2} E\right)^{2}-4 \lambda^{2} D^{2}-2 \lambda A^{\prime}$.

We first show that, if $\|A\|_{\infty}-2 \hat{K}_{A}>\left\|A^{\prime}\right\|_{\infty} / 2|\lambda|$, range $\left(Q_{\lambda}\right)_{0}$ is closed. The conclusion will eventually follow from this fact. 
If range $\left(Q_{\lambda}\right)_{0}$ is not closed, there is a sequence $f_{n}$ in $C_{0}^{\infty}(0, \infty)$, with $\left\|f_{n}\right\|=1$, and $\left\|Q_{\lambda} f_{n}\right\|$ converging to zero, since otherwise $\left(\left(Q_{\lambda}\right)_{R}\right)^{-1}$ would be a bounded linear transformation from range $\left(Q_{\lambda}\right)_{R}$ into $L_{2}^{n}[0, \infty)$, and therefore the same would be true of $\left(\left(Q_{\lambda}\right)_{0}\right)^{-1}$. Since $\left(Q_{\lambda}\right)_{0}$ is a closed operator, if $\left(\left(Q_{\lambda}\right)_{0}\right)^{-1}$ were continuous, range $\left(Q_{\lambda}\right)_{0}$ would be closed.

Since $\left(Q_{\lambda} f_{n}, Q_{\lambda} f_{n}\right)$ converges to zero, and since $\left(Q_{\lambda} f_{n}, Q_{\lambda} f_{n}\right)=$ $\left(\left(Q_{\lambda}\right)^{+} Q_{\lambda} f_{n}, f_{n}\right)$, it follows from our formula for $(Q)_{\lambda}^{+} Q_{\lambda}$ that $\left(\left(-\lambda^{2} D^{2}-2 \lambda A^{A}\right) f_{n}, f_{n}\right)$ becomes eventually less than any positive number as $n$ becomes large, since $\left(\left(D^{2}+A+\lambda^{2} E\right)^{2} f_{n}, f_{n}\right) \geq 0$. Thus, for any $\epsilon>0$, as $n$ becomes large, $4 \lambda^{2}\left\|D f_{n}\right\|^{2}$ becomes less than $2|\lambda|\left\|A^{\prime}\right\|_{\infty}+\epsilon$, and $\left\|D f_{n}\right\|^{2}$ becomes less than $\left\|A^{\prime}\right\|_{\infty} / 2|\lambda|-\epsilon / 4 \lambda^{2}$.

Let $\lambda_{0}$ be a nonnegative real number such that $\left\|A-\lambda_{0} E\right\|_{\infty}=K_{A^{*}}$. Then, for any $\epsilon>0$, when $n$ is large,

$$
\begin{aligned}
\left(\left(D^{2}+A\right) f_{n}, f_{n}\right) & =-\left\|D f_{n}\right\|^{2}+\left(A f_{n}, f_{n}\right) \\
& \geq-\left\|A^{\prime}\right\|_{\infty} / 2|\lambda|-\epsilon / 4 \lambda^{2}+\left(\left(A-\lambda_{0} E\right) f_{n}, f_{n}\right)+\lambda_{0}\left(f_{n}, f_{n}\right) \\
& \geq-\left\|A^{\prime}\right\|_{\infty} / 2|\lambda|-\epsilon / \lambda^{2}-K_{A}+\lambda_{0} .
\end{aligned}
$$

Since $\left\|Q_{\lambda} f_{n}\right\|$ converges to zero, it follows that $\left(\left(D^{2}+A+\lambda^{2} E\right) f_{n}, f_{n}\right)$ converges to zero, since $2 \lambda\left(D f_{n}, f_{n}\right)$ has real part zero. However, since for any $\epsilon>0$,

$$
\left(\left(D^{2}+A+\lambda^{2} E\right) f_{n}, f_{n}\right) \geq \lambda_{0}+\lambda^{2}-\left\|A^{\prime}\right\|_{\infty} / 2|\lambda|-\epsilon / \lambda^{2}-K_{A}
$$

when $n$ is large, we have a contradiction, provided that $\lambda_{0}+\lambda^{2}-\left\|A^{\prime}\right\|_{\infty} / 2|\lambda|$ $-K_{A}>0$. But $\lambda_{0} \geq\|A\|_{\infty}-K_{A}$. Thus, if $\delta$ is a positive real number with $\|A\|_{\infty}+\delta^{2}>\left(\left\|A^{\prime}\right\|_{\infty} / 2 \delta\right)+2 K_{A}$, range $\left(Q_{\lambda}\right)_{0}$ is closed when $|\lambda| \geq \delta$.

Now, if range $\left(Q_{\lambda}\right)_{0}$ is closed, range $\left(Q_{\lambda}\right)_{M}$ is closed, since $\left(Q_{\lambda}\right)_{M}$ is a $2 n$ dimensional extension of $\left(Q_{\lambda}\right)_{0}$, and since a finite dimensional extension of a closed subspace is closed. Furthermore, since $\left(\left(Q_{\lambda}\right)_{M}\right)^{*}$ is $\left(Q_{-\lambda}\right)_{0}$, and since any $f$ in domain $\left(Q_{-\lambda}\right)_{0}$ has $f(0)=D f(0)=0$, it follows that, when range $\left(Q_{\lambda}\right)_{0}$ is closed, $\left(Q_{\lambda}\right)_{M}$ is surjective, and therefore index $\left(Q_{\lambda}\right)_{M}=$ nullity $\left(Q_{\lambda}\right)_{M}$.

Since each $\left(Q_{\lambda}\right)_{M}$ is a Fredholm operator, it follows from Theorem 4 that index $\left(Q_{\lambda}\right)_{M}$ is constant on the set of all $\lambda \geq \delta$ and on the set of all $\lambda \leq-\delta$, although the two constants may be different.

Since $A$ is bounded, there is a $\lambda$ so large that $e^{-\lambda t} f(t)$ is in $L_{2}^{n}[0, \infty)$ for all solutions $f$ to the equation $\left(D^{2}+A\right) f=0$. But, if $Q_{\lambda} g=0$, then $\left(D^{2}+A\right)\left(e^{\lambda t} g(t)\right)=0$, so $g(t)=e^{-\lambda t} f(t)$, with $\left(D^{2}+A\right) f=0$. Thus, for $\lambda$ 
large, nullity $\left(Q_{\lambda}\right)_{M}=2 n$. Therefore, for all $\lambda \geq \delta$, nullity $\left(Q_{\lambda}\right)_{M}=2 n$. Since $\left(Q_{\lambda}\right)^{+}=Q_{-\lambda}$, it follows from Theorem 3 that nullity $\left(Q_{\lambda}\right)_{M}=0$ for all $\lambda \leq-\delta$. Thus, for any $\lambda \geq \delta, e^{\lambda t} f(t)$ is not in $L_{2}^{n}[0, \infty)$ for any solution $f$ to the equation $\left(D^{2}+A\right) f=0$, but $e^{-\lambda t} f(t) \in L_{2}^{n}[0, \infty)$. All that is required to complete the proof is to show that, if $\left(D^{2}+A\right) f=0, e^{-\delta t} f(t)$ and $e^{-\delta t} f^{\prime}(t)$ converge to zero as $t$ approaches infinity.

Since $e^{-\delta t} f(t)$ is in $S$, it follows that $\left(e^{-\delta t} f(t)\right)^{\prime}$ is in $L_{2}^{n}[0, \infty)$. But for any function $h$, if $h$ and $h^{\prime}$ are in $L_{2}^{n}[0, \infty), h(t)$ converges to zero as $t$ approaches infinity. Thus, in particular, $e^{-\delta t} f(t)$ converges to zero as $t$ approaches infinity.

Since $\left(e^{-\delta t} f(t)\right)^{\prime}$ is in $L_{2}^{n}[0, \infty)$, and since $e^{-\delta t} f(t)$ is also in $L_{2}^{n}[0, \infty)$, it follows that $e^{-\delta t} f^{\prime}(t)$ is in $L_{2}^{n}[0, \infty)$. It is then easy to show that $\left(e^{-\delta t} f^{\prime}(t)\right)^{\prime}$ is in $L_{2}^{n}[0, \infty)$, so that, by the above argument, $e^{-\delta t} f^{\prime}(t)$ converges to zero as $t$ approaches infinity. Theorem 5 is proved.

Theorem 6. Let $A$ be a continuously differentiable function from $[0, \infty)$ to the $n \times n$ complex Hermitian matrices. Suppose that both $A$ and $A^{\prime}$ are bounded. Let $\delta$ be a positive real number. Let $C$ be a positive real number such that $C^{2}<\delta$, and $\left(\delta-C^{2}\right)^{2}>2 C\left\|A^{\prime}\right\|_{\infty}$. Then, if $\|A\|_{\infty}+C^{2}>\left(\left\|A^{\prime}\right\|_{\infty} / 2 C\right)$ $+2 K_{A}$, there is a real number $\lambda$ with $|\lambda| \leq \delta$ such that $\left(D^{2}+A+\lambda E\right)_{0}$ does not have closed range.

Remark. Let $\delta$ and $\alpha$ be positive real numbers. Let $C$ be as above. Then, if $\alpha+C^{2}>\|A\|_{\infty}+\left(\left\|A^{\prime}\right\|_{\infty} / 2 C\right)+2 K_{A}$, it follows that

$$
\|A+\alpha E\|_{\infty}+C^{2} \geq \alpha+C^{2}-\|A\|_{\infty}>\left(\left\|A^{\prime}\right\|_{\infty} / 2 C\right)+2 K_{A} .
$$

Since $K_{A+a E} \leq K_{A}$, for $\alpha \geq 0$, it follows that if $\alpha+C^{2}>\|A\|_{\infty}+\left(\left\|A^{\prime}\right\|_{\infty} / 2 C\right)$ $+2 K_{A}$, there is a $\lambda$ with $|\lambda| \leq \delta$ such that $\left(D^{2}+A+\alpha E+\lambda E\right)_{0}$ does not have closed range.

Remark. It is well known that, for any $\lambda$ such that $\left(D^{2}+A+\lambda E\right)_{0}$ does not have closed range, $-\lambda$ must be a point of the essential spectrum of any selfadjoint extension of $\left(D^{2}+A\right)_{0}$.

Remark. If range $\left(D^{2}+A+\lambda E\right)_{0}$ is not closed, then the range of its adjoint operator $\left(D^{2}+A+\lambda E\right)_{M}$ is not closed. Therefore there is a $g$ in $L_{2}^{n}[0, \infty)$ for which there is no $f$ in $L_{2}^{n}[0, \infty)$ such that $\left(D^{2}+A+\lambda E\right) f=g$.

Proof of Theorem 6. Suppose that $H$ is the selfadjoint extension of $\left(D^{2}+A\right)_{0}$ formed by restricting $\left(D^{2}+A\right)_{M}$ to the set of all $f$ in $S$ with $f(0)=0$. Since $H$ is a finite dimensional extension of $\left(D^{2}+A\right)_{0}$, and since $\left(D^{2}+A\right)_{M}$ is a finite dimensional extension of $H$, and since, for any differ- 
ential expression $L$, range $\left(L_{0}+\lambda I\right)$ is closed if and only if the range of its adjoint $\left(L^{+}\right)_{M}+\lambda I$ is closed, where $\lambda$ is real, it follows that range $H+\lambda I$ is closed if and only if range $\left(D^{2}+A+\lambda E\right)_{0}$ is closed, since a finite dimensional extension of a closed subspace is closed.

Suppose that $[-\delta, \delta]$ contains no points $\lambda$ such that $-\lambda$ is in the essential spectrum of $H$. Since the essential spectrum of $H$ is the set of all cluster points of the spectrum of $H$, it follows that $[-\delta, \delta]$ contains only finitely many points $\lambda$ such that $-\lambda$ is in the spectrum of $H$. Let $P$ denote the orthogonal complement of the set of eigenvectors of $H$ corresponding to these eigenvalues. (Any point of the spectrum of $H$ which is not in the essential spectrum is an isolated point and therefore an eigenvalue.) Note that $P^{\perp}$ is finite dimensional. Let $H_{1}$ be the restriction of $H$ to $P$. $H_{1}$ is a selfadjoint operator taking the Hilbert space $P$ into itself. It follows from the spectral theorem that $\left\|H_{1} f\right\| \geq \delta\|f\|$ for every $f$ in domain $H_{1}$.

Let $Q_{\lambda}$ denote the differential expression $D^{2}+2 \lambda D+\lambda^{2} E+A$, for any real number $\lambda$. Note that, for any twice differentiable $f$,

$$
Q_{\lambda} f=e^{-\lambda t}\left(D^{2}+A\right)\left(e^{\lambda t} f\right)
$$

For any four times differentiable $f$,

$$
\left(Q_{\lambda}\right)^{+} Q_{\lambda} f=\left(D^{2}+A+\lambda^{2} E\right)^{2} f-4 \lambda^{2} D^{2} f-2 \lambda A^{\prime} f
$$

If $f$ is in domain $H^{2},\left(Q_{\lambda} f, Q_{\lambda} f\right)=\left(\left(Q_{\lambda}\right)^{+} Q_{\lambda} f, f\right)$, which follows from integration by parts, using the fact that $Q_{\lambda} f$ is in $S$ and $\left(Q_{\lambda} f\right)(0)=0$. Thus, if $f$ is in domain $\left(H_{1}\right)^{2}$,

$$
\left\|Q_{\lambda} f\right\|^{2} \geq\left(\delta-\lambda^{2}\right)^{2}\|f\|^{2}+4 \lambda^{2}\|D f\|^{2}-2|\lambda|\left\|A^{\prime}\right\|_{\infty}\|f\|^{2} .
$$

If $2|\lambda|\left\|A^{\prime}\right\|_{\infty}<\left(\delta-\lambda^{2}\right)^{2}$, we see that $\left\|Q_{\lambda} f\right\| \geq \epsilon>0$ for each $f$ in domain $\left(H_{1}\right)^{2}$ such that $\|f\|=1$, with $\epsilon=\left(\delta-\lambda^{2}\right)^{2}-2|\lambda|\left\|A^{\prime \prime}\right\|_{\infty}$.

We wish to show that the restriction $\left(Q_{\lambda}\right)_{1}$ of $Q_{\lambda}$ to domain $H_{1}$ has closed range in $L_{2}^{n}[0, \infty)$. It is easy to see that $\left(Q_{\lambda}\right)_{1}$ is a closed operator, so we will have showed the range is closed provided we can show that, for every $f$ in domain $H_{1}$ such that $\|f\|=1,\left\|Q_{\lambda} f\right\|^{2} \geq \epsilon$, where $\epsilon$ is, as above, $\left(\delta-\lambda^{2}\right)^{2}-2|\lambda|\left\|A^{\prime}\right\|_{\infty}$. We have already seen that the inequality holds provided that $f$ is in domain $\left(H_{1}\right)^{2}$. However, the closure of the restriction of $H_{1}$ to domain $\left(H_{1}\right)^{2}$ is $H_{1}$, as is the case for any selfadjoint operator. It is easy to see from this that the closure of the restriction of $Q_{\lambda}$ to domain $\left(H_{1}\right)^{2}$ is $\left(Q_{\lambda}\right)_{1}$. Thus the inequality holds for all $f$ in domain $H_{1}$, and therefore range $\left(Q_{\lambda}\right)_{1}$ is closed. 
Since $H$ is a finite dimensional extension of $H_{1}$, the restriction of $Q_{\lambda}$ to domain $H$ is a finite dimensional extension of $\left(Q_{\lambda}\right)_{1}$. Since $\left(Q_{\lambda}\right)_{M}$ is in turn a finite dimensional extension of the restriction of $Q_{\lambda}$ to domain $H$, it follows from the fact that a finite dimensional extension of a closed subspace is closed that, when range $\left(Q_{\lambda}\right)_{1}$ is closed, range $\left(Q_{\lambda}\right)_{M}$ is closed. Thus, when $\left(\delta-\lambda^{2}\right)^{2}-2|\lambda|\left\|A^{\prime}\right\|_{\infty}>0$, range $\left(Q_{\lambda}\right)_{M}$ is closed。 In other words, range $\left(Q_{\dot{\lambda}}\right)_{M}$ is closed whenever $|\lambda|<C$.

Now, using Theorem 4 as in the proof of Theorem 5, we see that nullity $\left(Q_{\lambda}\right)_{M}$ is constant on the set of $\lambda$ with $|\lambda|<C$. Since $\left(Q_{\lambda}\right)^{+}=Q_{-\lambda}$, and since nullity $\left(Q_{\lambda}\right)_{M}+$ nullity $\left(\left(Q_{\lambda}\right)^{+}\right)_{M}=2 n$, it follows that this constant nullity is $n$. Thus, since $Q_{\lambda} f=0$ if and only if $f=e^{-\lambda t} g$, with $\left(D^{2}+A\right) g=0$, we see that there is an $n$ dimensional subspace $K$ of solutions of $\left(D^{2}+A\right) g=0$ such that, for $g$ in $K, e^{\lambda t} g(t)$ is in $L_{2}^{n}[0, \infty)$, and for $g$ not in $K, e^{-\lambda t} g(t)$ is not in $L_{2}^{n}[0, \infty)$, for any $0 \leq \lambda<C$.

However, if $\|A\|_{\infty}+b^{2}>\left(\left\|A^{\prime}\right\|_{\infty} / 2 b\right)+2 K_{A}$, for some $b>0$, we know from Theorem 5 that $e^{-b t} g(t)$ is in $L_{2}^{n}[0, \infty)$ for any solution $g$ to the equation $\left(D^{2}+A\right) g=0$. If $\|A\|_{\infty}+C^{2}>\left(\left\|A^{\prime}\right\|_{\infty} / 2 C\right)+2 K_{A}$, select a positive number $b$ slightly smaller than $C$ so that the inequality remains true. We have just shown that, since $b<C$, there are solutions $g$ to the equation $\left(D^{2}+A\right) g=0$ with $e^{-b t} g(t)$ not in $L_{2}^{n}[0, \infty)$. However, this contradicts Theorem 5. The contradiction arose from assuming that, for all $\lambda$ with $|\lambda| \leq$ $\delta,\left(D^{2}+A+\lambda E\right)_{0}$ had closed range. Thus Theorem 6 is proved.

\section{REFERENCES}

1. N. Dunford and J. T. Schwartz, Linear operators. II: Spectral theory. Selfadjoint operators in Hilbert space, Wiley, New York, 1963. MR 32 \#6181.

2. S. Goldberg, Unbounded linear operators: Theory and applications, McGrawHill, New York, 1966. MR 34 \#580.

3. P. Hartman and C. Putnam, The gaps in the es sential spectra of wave equations, Amer. J. Math. 72 (1950), 849-862. MR 12, 414.

4. R. M. Kauffman, On the growth of solutions in the oscillatory case, Proc. Amer. Math. Soc. 51 (1975), 49-54.

DEPARTMENT OF MATHEMATICS AND COMPUTER SCIENCE, WESTERN WASHINGTON STATE COLLEGE, BELLINGHAM, WASHINGTON 98225 